\title{
Reliability of cavernous sinus sampling in management of Cushing's disease
}

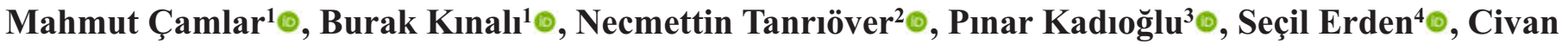 \\ Işlak $^{5} \odot$, Naci Koçer ${ }^{5} \odot$, Osman Kızılkılıç ${ }^{\oplus}$, Meryem Merve Ören ${ }^{6} \odot$, Nurperi Gazioğlu ${ }^{7}$
}

\begin{abstract}
${ }^{1}$ Department of Neurosurgery, University of Health Sciences, İzmir Tepecik Training and Research Hospital, İmir, Turkey ${ }^{2}$ Department of Neurosurgery, İstanbul University-Cerrahpaşa, Cerrahpaşa School of Medicine, Istanbul, Turkey

${ }^{3}$ Department of Internal Medicine, Division of Endocrinology-Metabolism and Diabetes, İstanbul University-Cerrahpaşa, Cerrahpaşa School of Medicine, İstanbul, Turkey

${ }^{4}$ Department of Fundamentals of Nursing, Istanbul University-Cerrahpaşa, Florance Nightingale Faculty of Nursing, Istanbul, Turkey ${ }^{5}$ Department of Neuroradiology, İstanbul University-Cerrahpaşa, Cerrahpaşa School of Medicine, Istanbul, Turkey

${ }^{6}$ Department of Public Health Services Presidency, Erzurum Provincial Health Directorate, Erzurum, Turkey

${ }^{7}$ Department of Neurosurgery, İstanbul Bilim University School of Medicine, Istanbul, Turkey
\end{abstract}

\section{ABSTRACT}

Objectives: The purpose of this study is to find out the accuracy of bilateral cavernous sinus sampling (CSS) for preoperative tumor lateralization within the pituitary in Cushing's disease (CD).

Methods: Sixty-five patients who had undergone transsphenoidal surgery (TSS) following CSS for CD between 2000-2016 at our institution were analyzed retrospectively. All patients underwent bilaterally CSS with corticotropin-releasing hormone $(\mathrm{CRH})$ stimulation. Radiological, preoperative, and pathological findings with remission status were correlated with CSS data. The accuracy of CSS is decided according to compliance with the magnetic resonance imaging (MRI), pathology or remission after surgery of the normal MRI cases.

Results: CSS indicated the correct lateralization in $52(80 \%)$ patients. There was the same level elevation of both sides in 3 patients who had central adenoma. Remission rate in the final follow-up was $83.87 \%$ for 65 patients. We found out the contralateral pathological side in $13(20 \%)$ of the patients showing false lateralization. Twenty-four patients with normal MRI had a positive accuracy rate of $83 \%$. Of those patients, with a positive accuracy of CSS sampling, $18(90 \%)$ had a positive remission. As a result of the Kappa analysis, statistically significance and relation was found between the final diagnosis and lateralization test $(\kappa=0.63 p$ $<0.001)$.

Conclusions: Our results showed that CSS is a safe and reliable method for microadenomas which has high diagnostic accuracy $(80 \%)$ in indicating the correct lateralization in $\mathrm{CD}$, providing us higher remission rates in a challenging pathology.

Keywords: Cushing's disease, pituitary tumor, adrenocorticotropic hormone, cavernous sinus sampling, venous sampling

$\mathrm{C}$ ushing's disease is a rare disease characterized by uncontrolled release of Adrenocorticotropic hormone $(\mathrm{ACTH})$ from the anterior pituitary and conse- quently excessive cortisol release from the adrenal gland. Diagnosis can be delayed by 2-4 years, especially in patients with mild or cyclic hypercortisolism 
$[1,2]$. Biochemical tests should be performed to consolidate the diagnosis with clinical findings due to hypercortisolism. After diagnostic tests, dexametasone supression, metyrapone or corticotropin releasing hormone $(\mathrm{CRH})$ stimulation test should be performed to distinguish the diagnose from ectopic corticotropin syndrome [3-5]. Although they are reliable tests, they are often inadequate to make this distinction. At this point, contrast enhanced pituitary magnetic resonance imaging (MRI) is used for the confirmation of presence or absence of a pituitary adenoma. The median size of the adenomas in Cushing's disease (CD) is 5 $\mathrm{mm}$. MRI with $3 \mathrm{~mm}$ sections can not capture approximetely $40-50 \%$ of the adenomas smaller than this median size and reported as normal $[6,7]$. When the MRI findings are uncertain or biochemical tests are inconclusive, subtotal or total removal of the pituitary gland may be performed despite the endocrine deficiency risk.

Central venous sampling is an assistive method used to localize the pathology $[8,9]$. Because of false positivity risk in laboratory tests it is a key method for definitive diagnosis and lesion localization. It can be performed in the inferior petrosal sinus or cavernous sinus [10]. It is possible to obtain an almost exact result with this method. If there is a difference $>1.4$ in ACTH levels between the sides in venous sampling, it is accepted that it predicts the side of the lesion in CD patients with negative MRI.

The primary objective of this study was to to find out the accuracy of bilateral CSS in preoperative tumor lateralization within the pituitary in patients with CD.

\section{METHODS}

\section{Study Design}

Our prior study with 26 patients was expanded and re-studied [11]. After the local Ethics Comittee aproval we retrospectively identified 65 consequtive patients who had undergone cavernous sinus sampling (CSS) and transsphenoidal surgery (TSS) in the department of neurosurgery, Cerrahpaşa School of Medicine, by a single surgeon (NG) between 2000 and 2016.

\section{Participants}

Patients who have endocrinological evidence of
CD had diagnosed with a standard protocol by a multidisciplinary team in Istanbul University Pituitary Center. All of the patients with normal MRI or lesions smaller than $6 \mathrm{~mm}$ who were operated via TSS approach were evaluated. Data of patients were collected from the medical history records. Endogenous hypercortisolism was confirmed with the increased urinary free cortisol (UFC), loss of circadian rhythm and lack of suppression of cortisol after low dose dexamethasone supression tests (DST). Pituitary origin was diagnosed with the $8 \mathrm{mg}$ dexamethasone test and CRH stimulation test. We included the patients with the MRI evaluations who showed up either a normal MRI or a mass smaller than $6 \mathrm{~mm}$ within the gland. All patients with CSS indicating central etiology underwent TSS without perioperative steroid coverage.

\section{Remission Criteria}

Remission was defined as: normalization of circadian rthym and ACTH and postoperative basal cortisol $<3.5 \mu \mathrm{g} / \mathrm{dl}$; serum cortisol $<1.8 \mu \mathrm{g} / \mathrm{dl}$ after $1 \mathrm{mg}$ dexamethasone suppression test.

\section{Statistical Analysis}

Continuous variables are reported as means \pm standard deviation and categorical variables are reported as percentages. Student's t test was used for comparison of normal distributed variables and MannWhitney U test was used for non-normally distributed variables. Categorical variables were compared by the Chi-Square test or Fisher's exact test as appropriate. Kappa coefficient was used for a statistical measure of inter-rater reliability. A p value less than 0.05 were considered significant.

\section{RESULTS}

\section{Demographics}

Retrospective analysis of 65 patients who were diagnosed as Central Cushing's Disease showed female dominancy of $81.53 \%$. There were 12 male patients and 53 female patients. Mean age was 38.78 years (ranging from 5-66). Four (6.15\%) of the patients were under 18 years.

\section{Preoperative MRI}

All patients underwent preoperative MRI evalua- 


\section{MRI Findings}

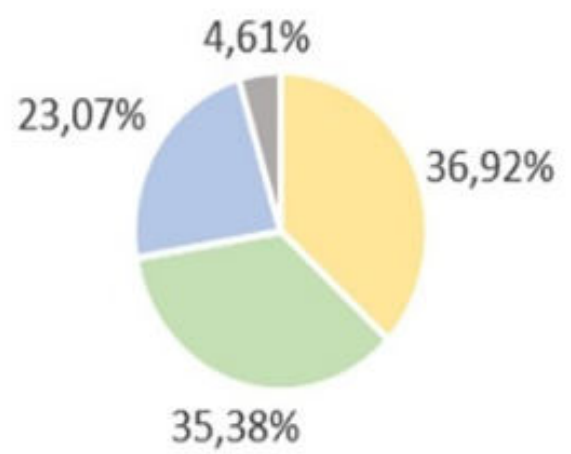

Negative Pituitary MRI

Microadenoma on right side
Microadenoma on left side

- Microadenoma located midline

Fig. 1. Distribution of patients according to MRI findings.

tion. All of the patients showed up normal MRI or microadenoma smaller then $6 \mathrm{~mm} .24(36.92 \%)$ of them were with normal MRI, 23 (35.38\%) with left sided microadenoma, $15(23.07 \%)$ with right sided microadenoma and $3(4.61 \%)$ of them were central microadenoma (Fig.1).

\section{CSS}

Sixty-five patients underwent CSS under general anesthesia or sedation. Heparinization with 5000 UI bolus followed by $1000 \mathrm{U} / \mathrm{h}$ additional dose has been done. Bifemoral punction with 5F catheter, Picard (Cook) catheter to IPS (Inferior petrosal sinus) followed by 0.18 microcatheter placement to CS (cavernous sinus) by 0,16 guide. Blood samples were taken without $\mathrm{CRH}$ and in the 1st, 2nd, 5th and 10th minutes after $\mathrm{CRH}$ stimulation simultaneously from both cavernous sinüs (CS). The central / peripheral (c $/ p)$ ratio was interpreted as central if the gradient was greater than 3 with $\mathrm{CRH}$ stimulation and we evaluated $\mathrm{R} / \mathrm{L}$ or $\mathrm{L} / \mathrm{R}$ ratio as lateralization if it was great equal 1.4. A positive gradient between right and left sides was found in 62 patients ranging from 1.56 to 250 (mean: 19.37$)$. There was $3(4.61 \%)$ without lateralization but central to peripheral gradient (Table 1). We found accuracy of $80 \%(52 / 65)$ which was decided as positive if the CSS side is compatible with the MRI, pathology or remission after hemihypophysectomy of the normal MRI cases.

Thirteen patients showed false CSS lateralization. Eleven patients among this 13 false lateralization were in remission. Of the 24 patients with normal MRI, 20

Table 1. Data forpatients who underwent CSS and TSS, remission rate, accuracy

\begin{tabular}{|c|c|c|c|c|c|c|c|}
\hline \multicolumn{6}{|c|}{ TSS exploration findings $n=65^{*}$} & \multirow[b]{2}{*}{$\begin{array}{l}\text { Final follow- } \\
\text { up remission }\end{array}$} & \multirow[b]{2}{*}{ Accuracy* } \\
\hline CSS ratio & No & Same side $\mathrm{HH}$ & $\begin{array}{c}\text { Same side } \\
\text { A }\end{array}$ & $\begin{array}{c}\text { Counter } \\
\text { A }\end{array}$ & $\mathrm{HH}+\mathrm{A}$ & & \\
\hline Right & 31 & 8 & 4 & 3 & 16 & 27 & 21 \\
\hline Left & 31 & 9 & 15 & 6 & 1 & 25 & 28 \\
\hline Equal & 3 & 0 & 3 & 0 & 0 & 3 & 3 \\
\hline
\end{tabular}




\section{Type of surgery}

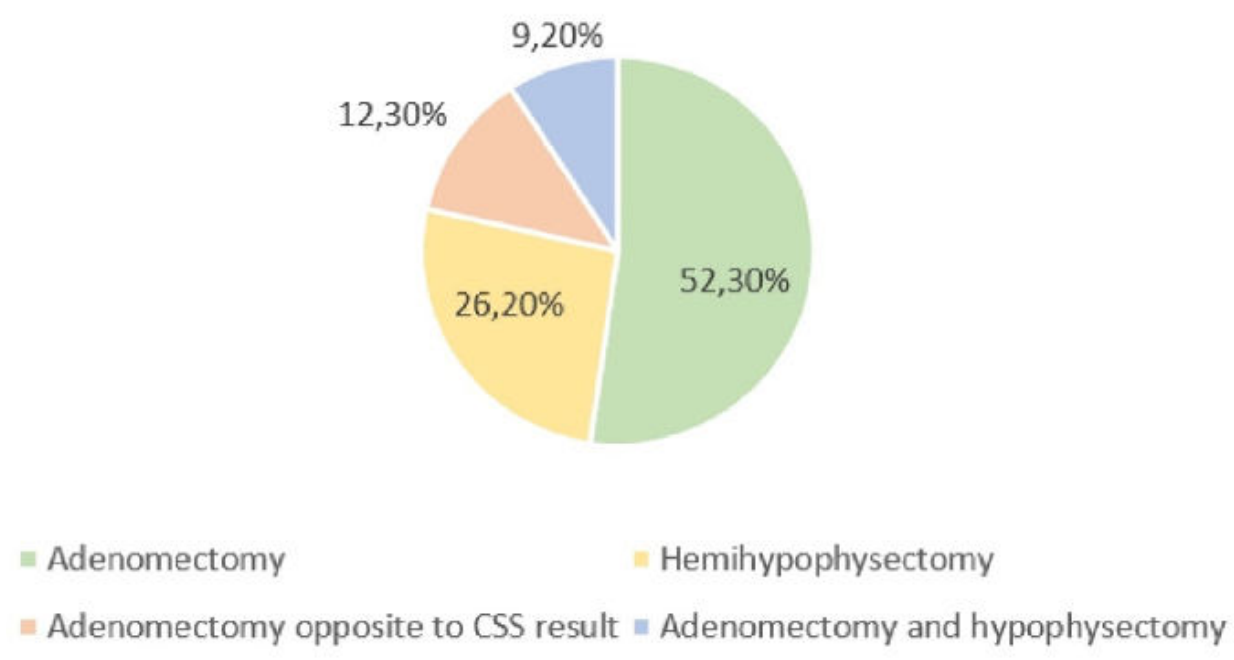

Fig. 1. Type of surgery.

had positive accuracy for CSS (83\%). Patients with a positive accuracy of CSS sampling had a remission rate of 18/20 (90\%). Tumors less than $6 \mathrm{~mm}$ which was verified by MRI scans had an accuracy of 31/41 $(75.6 \%)$. Twenty-six of them had a positive remission in the final follow-up (83.87\%).

There was only one complication of guidewire breakage during cavernous sinus sampling. The distal part of the wire had to be left but removed during the following TSS after the removal of tumor. There were no other complications.

\section{Surgical approach and remission}

We performed either endoscopic or microscopic TSS for all of the cases after CSS. We started to use endoscopic TSS since 2007 and all patients were operated endoscopically after 2007. Surgical strategy consisted of exploring the whole gland, starting from the suspected side. If the adenoma is detected, adenomectomy is performed. If not hemihypophysectomy was performed according to the CSS lateralization. There were 17 temporary diabetes insipidus and 1 permenant diabetes insipidus treated with desmopressin.

\section{Pathology}

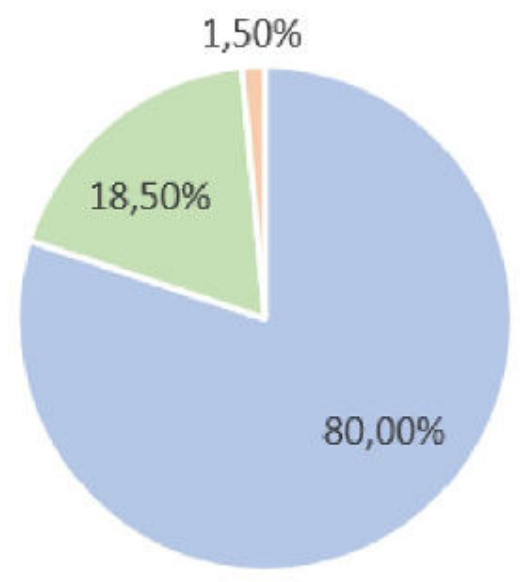

= ACTH $(+)$ Adenoma = Adenohypophysis = Hyperplasia

Fig. 1. Distribution of the pathological result of the patients. 
One patient had rhinorrhea who was treated by lomber CSF drainage of 72 hours. Four patients had hypothyroidism post-operatively and 1 patient with panhypopituitarism who underwent medical treatment. One of the patients had a menenjitis treated by antibiotic treatment. There were no mortality related to our surgical procedure but 4 patients died during long-term follow-up because of complications related to $\mathrm{CD}$. We had no mortality nor neurological morbidity after the surgical procedures. Patients who had remission on the final follow up was 55 (84.6\%). Types of surgeries performed and pathological results of the patients are as shown in Figs. 2 and 3.

\section{DISCUSSION}

Treatment of Cushing's disease is problematic and should be treated by a multidisciplinary team and the treatment aims to improve the patients clinical manifestations with the resection of tumoral mass normalizing the hormone secretion. Despite all treatment methods, the biggest problem with these patients is the inability to achieve the desired remission rates and the recurrence of the disease. There are considerable number of CD patients with normal MRI, with empty sella, and with some suspect lesions or fullness in one side of the pituitary. Preoperative MRI identification of an adenoma has been associated with high remission rates [12]. Of course this can be linked to the advantages of knowing the enemy and dealing with it. Invisible adenomas are the adenomas that surgeons are mostly not like to operate. Endoscopic TSS may be a good option for better visualization of adenomas but most of the time it doesn't solve this suspect lesion problem completely.

In 1985 Oldfield et al. [13] reported the high accuracy of inferior petrosal sinus sampling (IPSS) method in differentiating the ectopic sourced Cushing's syndrome from pituitary adenomas. In time, venous sampling methods began to spread and a number of series began to be published. The common feature of the series was that the methods of venous sampling either IPSS or CSS were helpful to identify the central etiology in ACTH-dependent CD. There is however no consensus about their value of correctly locating the tumor within the pituitary $[12,14-16,17]$. The trick point is the experience of the interventional neuroradiology team. This study is not a comparison between IPSS and CSS, however it is worth to review the advantages and disadvantages of both methods (Table 2). ACTH secreting pituitary adenomas are mostly located laterally [18]. Therefore, venous sampling results are one of the most indispensable guide for surgeon's intervention. Both halves of the pituitary gland drain into the cavernous sinus of its own side, and therefore direct cavernous sinus sampling is thought to yield more accurate results about lateralization of the adenoma because of the high concentration of the ACTH levels $[12,17]$. Whether it is CSS or IPSS, the placement of catheters in both is very important for the interpreting of lateralization results [19].

In our study CSS sampling had a $80 \%$ accuracy.

Teramoto et al. [14] reported the correct lateralization of CSS $57 \%$ to $80 \%$. Of the 24 patients whose MRI showed no adenoma, we found correct lateralization for 20 (sensitivity 83\%). Patients with a positive accuracy of CSS sampling had a remission rate of $18 / 20(90 \%)$. Of the 41 patients who has an adenoma $<6 \mathrm{~mm}$ on their MRI, correct lateralization for $31 / 41$ $(75.6 \%)$ was found which 26 of them had a final remission in the follow-up (83.87\%). Of course there are cases where CSS is also insufficient. In our study we found false CSS result for 13 patients. In this group, 4 patients with normal MRI had an contralateral ade-

\section{Table 2. Advantages and disadvantages of CSS and IPSS}

\begin{tabular}{lcc}
\hline & Advantages & Disadvantages \\
\hline IPSS & Easier & Frequent venous variations \\
& Not reliable for lateralization \\
CSS & $\begin{array}{c}\text { Closer to the lesion, providing } \\
\text { more elevated ACTH concentration in the } \\
\text { sample }\end{array}$ & $\begin{array}{c}\text { Need for experienced neuroradiologist } \\
\text { Painful procedure requiring general anesthesia or } \\
\text { sedation }\end{array}$ \\
\hline
\end{tabular}

$\mathrm{CSS}=$ Cavernous sinus sampling, IPSS $=$ Inferior petrosal sinus sampling, $\mathrm{ACTH}=$ Adrenocorticotropic hormone 
noma compared to CSS sampling side.

Incorrect (false) lateralization is a possible problem in all venous sampling methods. There may be many reasons for this undesirable situation. Mamelak et al. [20] emphasized that the asymmetric drainage of the inferior petrosal and cavernous sinuses was the main cause of the incorrect lateralization. Variations of the venous system, communications between cavernous sinuses and the compartmantalizations of the sinuses with fibrous septaes are the other possible reasons for this false lateralizations [17, 20,21].

When we look at the literature, we see that our results are compatible with other studies. Correct rate of CSS sampling was reported as $82 \%$ by Burkhardt et al. [8], $91 \%$ by Teramoto et al. [14, 17] and $83 \%$ by Graham et al. [21]. In our institution we prefer CSS for the preoperative assessment of lateralization. It is useful to underline again that the method of sampling is determined according to the experience of the neuroradiology team. Surgery may affect the venous drainage therefore CSS may not be helpful in relapsed cases. All cases in this study were operated first time. The accuracy of the CSS in recurrent cases may be the subject of further studies.

\section{CONCLUSION}

Nowadays, adenomas are detected in smaller sizes due to the widespread use of MRI for various reasons. The biggest problem for invisible adenomas and small sized adenomas is finding adenoma during surgery or where to perform the hemihypophysectomy. Every additional information is helpful to the surgeon therefore we emphasize that performing CSS may improve the remission rates.

\section{Conflict of interest}

The authors disclosed no conflict of interest during the preparation or publication of this manuscript.

\section{Financing}

The authors disclosed that they did not receive any grant during conduction or writing of this study.

\section{Acknowledgement}

All authors certify that they have no affiliations with or involvement in any organization or entity with any financial interest or non-financial interest in the subject matter or materials discussed in this manuscript. This article does not involve any studies with human participants performed by any of the authors nor does it contain any studies with animals.

\section{REFERENCES}

1. Etxabe J, Vazquez. Morbidity and mortality in Cushing's disease: an epidemiological approach. JA Clin Endocrinol (Oxf) 1994;40:479-84.

2. Valassi E, Santos A, Yaneva M, Tóth M, Strasburger CJ, Chanson P, et al. The European Registry on Cushing's syndrome: 2year experience. Baseline demographic and clinical characteristics. ERCUSYN Study Group. Eur J Endocrinol 2011;165:383-92.

3. Sasaki Y, Katabami T, Asai S, Fukuda H, Tanaka Y. In the overnight dexamethasone suppression test, $1.0 \mathrm{mg}$ loading is superior to $0.5 \mathrm{mg}$ loading for diagnosing subclinical adrenal Cushing's syndrome based on plasma dexamethasone levels determined using liquid chromatography-tandem mass spectrometry. Endocr J 2017;30:833-42.

4. Daniel E, Aylwin S, Mustafa O, Ball S, Munir A, Boelaert K, et al. Effectiveness of metyrapone in treating Cushing's syndrome: a retrospective multicenter study in 195 patients. J Clin Endocrinol Metab 2015;100:4146-54.

5. Nieman LK, Oldfield EH, Wesley R, Chrousos GP, Loriaux DL, Cutler GB. A simplified morning ovine corticotropin releasing hormone stimulation test for the differential diagnosis of adrenocorticotropin-dependent Cushing's syndrome. J Clin Endocrinol Metab 1993;77:1308-12.

6. Grober Y, Grober H, Wintermark M, Jane JA Jr, Oldfield EH. Comparison of MRI techniques for detecting microadenomas in Cushing's disease. J Neurosurg 2018;128:1051-7.

7. Bansal V, El Asmar N, Selman WR, Arafah BM. Pitfalls in the diagnosis and management of Cushing's syndrome. Neurosurg Focus 2015;38:E4.

8. Burkhardt T, Flitsch J, van Leyen P, Sauer N, Aberle J, Grzyska U, et al. Cavernous sinus sampling in patients with Cushing's disease. Neurosurg Focus. 2015;38:E6.

9. Kai Y, Hamada J, Nishi T, Morioka M, Mizuno T, Ushio Y. Usefulness of multiple site venous sampling inthe treatment of adrenocorticotropic hormone producing pituitary adenomas. Surg Neurol 2003;59:292-98.

10. Flitsch J, Lüdecke DK, Knappe UJ, Grzyska U. Cavernous sinus sampling in selected cases of Cushing's disease. Exp Clin Endocrinol Diabetes 2002;110:329-35.

11. Gazioglu N, Ulu MO, Ozlen F, Albayram S, Islak C, Kocer $\mathrm{N}$, et al. Management of Cushing's disease using cavernous sinus sampling: effectiveness in tumor lateralization. Clin Neurol Neurosurg 2008;110:333-8.

12. Doppman JL, Nieman LK, Chang R, Yanovski J, Cutler GB $\mathrm{Jr}$, Chrousos GP, et al. Selective venous sampling from the cavernous sinuses is not a more reliable technique than sampling from the inferior petrosal sinuses in Cushing's syndrome. J Clin 
Endocrinol Metab 1995;80:2485-9.

13. Oldfield EH, Chrousos GP, Schulte HM, Schaaf M, McKeever PE, Krudy AG, et al. Preoperative lateralization of ACTHsecreting pituitary microadenomas by bilateral and simultaneous inferior petrosal venous sinus sampling. $N$ Engl J Med 1985;312:100-3.

14. Teramoto A, Nemoto S, Takakura K, Sasaki Y, Machida T. Selective venous sampling directly from cavernous sinüs in Cushing's syndrome. J Clin Endocrinol Metab 1993;76:637-41. 15. Oldfield EH, Doppman JL, Nieman LK, Chrousos GP, Miller DL, Katz DA, et al. Petrosal sinus sampling with and without corticotropin-releasing hormone for the differential diagnosis of Cushing's syndrome. New Eng J Med 1991;325:897-905.

16. Booth GL, Redelmeier DA, Grosman H, Kovacs K, Smyth HS, Ezzat S. Improved diagnostic accuracy of inferior petrosal sinus sampling over imaging for localizing pituitary pathology in patients with Cushing's disease. J Clin Endocrinol Metab 1998;83:2291-5.

17. Teramoto A, Yoshida Y, Sanno N, Nemoto S. Cavernous sinus sampling in patients with adrenocorticotrophic hormone-depen- dent Cushing's syndrome with emphasis on inter- and intracavernous adrenocorticotrophic hormone gradients. J Neurosurg 1998;89:762-8.

18. Fahlbusch R, Buchfelder M, Müller OA.Transsphenoidal surgery for Cushing's disease. J R Soc Med1986;79:262-9.

19. Liu C, Lo JC, Dowd CF, Wilson CB, Kunwar S, Aron DC, et al. Cavernous and inferior petrosal sinus sampling in the evaluation of ACTH-dependent Cushing's syndrome. Clin Endocrinol (Oxf) 2004;61:478-86.

20. Mamelak AN, Dowd CF, Tyrrell JB, McDonald JF, Wilson $\mathrm{CB}$. Venous angiography is needed to interpret inferior petrosal sinus and cavernous sinus sampling data for lateralizing adrenocorticotropin-secreting adenomas. J Clin Endocrinol Metab 1996;81:475-81.

21. Graham KE, Samuels MH, Nesbit GM, Cook DM, O'Neill OR, Barnwell SL et al. Cavernous sinus sampling is highly accurate in distinguishing Cushing's disease from the ectopic adrenocorticotropin syndrome and in predicting intrapituitary tumor location. J Clin Endocrinol Metab 1999;84:1602-10. 